Keywords: endometrial cancer; clinician preferences; adjuvant chemotherapy; decision-making; patient preferences; outcomes research

\title{
Patients' and clinicians' preferences for adjuvant chemotherapy in endometrial cancer: an ANZGOG substudy of the PORTEC-3 intergroup randomised trial
}

On behalf of the ANZGOG and PORTEC Group, Prunella Blinman*,1, Linda Mileshkin², Pearly Khaw ${ }^{2}$, Geraldine Goss ${ }^{3}$, Carol Johnson ${ }^{4}$, Anne Capp ${ }^{5}$, Susan Brooks ${ }^{6}$, Gerard Wain ${ }^{7}$, Ilka Kolodziej ${ }^{8}$, Anne-Sophie Veillard ${ }^{8}$, Rachel $\mathrm{O}^{\prime}$ Connell ${ }^{8}$, Carien L Creutzberg ${ }^{9}$ and Martin R Stockler ${ }^{1,8,10}$

${ }^{1}$ Concord Cancer Centre, Hospital Road, Concord, NSW 2139, Australia; ${ }^{2}$ Peter MacCallum Cancer Centre, St Andrews Place, East Melbourne, VIC 8006, Australia; ${ }^{3}$ Monash Medical Centre, 246 Clayton Road, Clayton, VIC 3168, Australia; ${ }^{4}$ Wellington Hospital, Riddiford St, Newtown, Wellington 6021, New Zealand; ${ }^{5}$ Calvary Mater Newcastle, Edith St \& Platt St, Waratah, NSW 2298, Australia; ${ }^{6}$ Auckland City Hospital, 2 Park Rd, Grafton, Auckland 1023, New Zealand; 'Westmead Hospital, Hawkesbury Road \& Darcy Road, Westmead, NSW 2145, Australia; ${ }^{8}$ NHMRC Clinical Trials Centre, Lifehouse Building, 119-143 Missenden Road, Camperdown, NSW 2050, Australia; ${ }^{9}$ Leiden University Medical Center, Albinusdreef 2, 2333 ZA Leiden, The Netherlands and ${ }^{10}$ University of Sydney, NSW 2006, Australia

Background: To determine the minimum survival benefits that patients, and their clinicians, judged sufficient to make adjuvant chemotherapy (ACT) worthwhile, in addition to pelvic radiotherapy, for women with high risk and advanced stage endometrial cancer.

Methods: Eighty-three participants in the PORTEC-3 trial completed a time trade-off questionnaire before and after adjuvant therapy; 44 of their clinicians completed it once only. The questionnaire used four hypothetical scenarios including baseline survival times without ACT of 5 and 8 years, and baseline survival rates at 5 years without ACT of 50 and $65 \%$.

Results: Over $50 \%$ of patients judged an extra 1 year of survival time or an extra $5 \%$ in survival rate sufficient to make ACT worthwhile. Over $50 \%$ of clinicians judged an extra 1 year of survival time, or an extra $10 \%$ in survival rate, sufficient to make ACT worthwhile. Compared with patients, clinicians required similar survival time benefits (medians both 1 year, $P=0.4$ ), but larger survival rate benefits (medians $8.5 \%$ vs $5 \%, P=0.03$ ), and clinicians' preferences varied less (IQR 0.5-1.5 years vs $0.4-2$ years, $P=0.0007 ; 5-10 \%$ vs $1-13 \%$, $P=0.004)$. Patients' preferences changed over time for the survival rate scenarios depending on whether they had ACT or not (change in median benefit - 3 months vs 2.5 months respectively, $P=0.028$ ). There were no strong predictors of patients' or clinicians' preferences.

Conclusions: Patients and clinicians judged moderate survival benefits sufficient to make ACT worthwhile after pelvic radiotherapy for endometrial cancer. These benefits are larger than those judged sufficient by patients with breast or colon cancers, but similar to those judged sufficient by patients with lung or ovarian cancers.

Endometrial cancer is the most common gynaecological cancer in developed countries (12.9 new cases per 1000000 women per year), and the second most common (after cervical cancer) in less developed countries (6.0 new cases per 100000 women per year) (Jemal et al, 2011). There were 320000 new cases of endometrial cancer and 76000 deaths from endometrial cancer worldwide in 2012 (Torre et al, 2015).

*Correspondence: Dr P Blinman; E-mail: prunella.blinman@sswahs.nsw.gov.au

Received 5 April 2016; revised 18 August 2016; accepted 10 September 2016; published online 20 October 2016

(c) 2016 Cancer Research UK. All rights reserved 0007-0920/16 
Surgery with a total hysterectomy and bilateral oophorectomy (TAH BSO) is the mainstay of treatment for endometrial cancer, but locoregional recurrence occurs in many patients. Adjuvant pelvic radiotherapy reduces the risk of locoregional recurrence, but does not improve overall survival (OS) in patients with early stage, high-intermediate risk endometrial cancer (Keys et al, 2004; Nout et al, 2011). Adjuvant chemotherapy (ACT) remains unproven despite its role suggested by the increased risk of developing distant metastases in patients with high-risk features, and the study of various strategies of incorporating ACT in the treatment of endometrial cancer in randomised trials (Morrow et al, 1990; Maggi et al, 2006; Randall et al, 2006; Susumu et al, 2008; Hogberg et al, 2010).

The PORTEC-3 trial was designed to test the addition of ACT to standard surgery and radiotherapy, by comparing surgery followed by pelvic radiotherapy alone $v s$ surgery followed by pelvic radiation with concurrent chemotherapy followed by four cycles of chemotherapy in patients with high-risk and advanced stage endometrial cancer. The potential benefits of ACT in the PORTEC-3 trial are improvements in DFS and OS at the cost of increased toxicity and inconvenience due to the longer and more intensive treatment.

An individual's preferences regarding ACT, such as that in the PORTEC-3 trial, reflect their personal value judgement about the trade-off between its benefits and harms. Studies of preferences for ACT quantify the minimum benefits judged sufficient to justify its possible harms and inconveniences (Blinman et al, 2012). Knowledge of a patient's preferences allows tailoring of communication and decision-making and is an important component of patientcentred care and shared decision-making.

Our studies of preferences in other cancers have shown that most patients judge small benefits sufficient to make ACT worthwhile in breast and colon cancers (e.g. median absolute benefits of $1 \%$ in survival rates) (Duric et al, 2005; Blinman et al, 2010), but that larger benefits were required to make ACT worthwhile in non-small-cell lung cancer (NSCLC), and intraperitoneal chemotherapy worthwhile in advanced ovarian cancer (e.g. median absolute benefits of $5 \%$ in survival rates) (Blinman et al, 2013, 2015). We were unable to find published data about preferences for ACT in endometrial cancer.

The aim of this sub-study of the PORTEC-3 trial was to determine the minimum survival benefits that participating patients and clinicians judged sufficient to make the addition of ACT worthwhile in women with endometrial cancer undergoing surgery and pelvic radiotherapy, and the factors influencing these preferences.

\section{MATERIALS AND METHODS}

Study design. The preferences study was as an observational cohort study designed and conducted by ANZGOG investigators, and nested within the PORTEC- 3 trial at the 16 Australian and New Zealand participating sites. Participants in the preferences substudy were patients participating in the PORTEC-3 trial at these sites, and their clinicians (medical oncologists, radiation oncologists, and gynaeoncological surgeons). Ethics approval of the preferences study was obtained from each participating centre.

In the PORTEC-3 trial, patients were randomised in a $1: 1$ ratio to either ACT in addition to pelvic radiotherapy (experimental group) or pelvic radiotherapy alone (control group). ACT comprised two cycles of cisplatin $50 \mathrm{mg} \mathrm{m}^{-2}$ given in the first and fourth week of pelvic radiotherapy, followed by four cycles of paclitaxel (175 $\mathrm{mg} \mathrm{m}^{-2}, 3 \mathrm{~h}$ infusion) and carboplatin (AUC 5) given once every 3 weeks. Pelvic radiotherapy in both arms was
48.6 Gy (1.8 Gy per fraction) with a brachytherapy boost for cervical invasion. The main efficacy results of the PORTEC-3 trial have not yet been published.

Preferences questionnaire. Patients' and clinicians' preferences for ACT were elicited with a self-completed paper questionnaire. Patients completed the questionnaire at baseline (after randomisation but before any trial treatment) and again at 9 months, which was about three months after finishing ACT in the experimental group. Clinicians completed the questionnaire once only.

The questionnaire used the time trade-off method to determine the minimum survival benefits judged sufficient to make the addition of ACT to pelvic radiotherapy worthwhile, as in our previous studies (Duric et al, 2005; Blinman et al, 2010, 2012, 2013, 2015). There were two different types of time trade-off questions based on standardised, hypothetical scenarios. The first type of question asked participants to consider adding extra times to a baseline survival time while the second type asked participants to consider adding extra chances of survival to a baseline survival rate. For example, in the first type of question the patient was asked to imagine a life expectancy of 5 years without chemotherapy or a longer life expectancy with ACT. The extra survival times due to ACT ranged from 1 day to 15 years. Each type of question had two different baseline prognoses (survival times of 5 or 8 years; survival rates at 5 years of 50 or $65 \%$ ) resulting in four hypothetical scenarios.

Other assessments. Other assessments included a study-specific questionnaire to record participant's age, marital status, occupation, smoking history, and level of social supports. Cancer histology, stage, and type of surgery were recorded as part of PORTEC-3 trial. The Patient Disease and Treatment Form (Patient DATA Form) (Stockler et al, 2007) was used to assess the patients ratings of their expected health-related quality of life (HRQL) during ACT, as in our previous preferences studies (Duric et al, 2005; Blinman et al, 2010, 2013, 2015). The Hospital Anxiety and Depression Scale (HADS) (Zigmond and Snaith, 1983) was used to assess the effect of symptoms of anxiety and depression on preferences at baseline, again as used in our previous preferences studies (Duric et al, 2005; Blinman et al, 2010, 2013, 2015).

Questionnaires were distributed to patients by a trial nurse at the baseline visit for the trial, after randomisation but before starting the randomly allocated ACT. Trial nurses were asked to go through the written instructions for the questionnaire with participants, but not to influence participants' responses. Questionnaires were distributed to clinicians after activation of the trial at their site.

Statistical analysis. This study was primarily descriptive and exploratory. The primary endpoint for descriptive purposes was the smallest benefit judged sufficient to make ACT worthwhile in each of the four scenarios. The preference data were expected to be highly skewed requiring robust methods used and validated in our previous studies (Duric et al, 2005; Blinman et al, 2010, 2013, 2015). The primary endpoint for comparisons between groups and for correlations with other factors was the average of the smallest benefits judged sufficient for the two survival time scenarios. Comparisons between groups were based on the non-parametric Kruskal-Wallis test. Determinants of preferences were assessed using multivariable linear regression after normal score transformation of the preferences data (Gibbons and Chakraborti, 2011). Logistic, ordinal, and mixture models were used to determine the sensitivity of the results to the methods of analysis, as in our previous studies. $P$-values were interpreted conservatively to account for the multiple comparisons: $P$-values from 0.01 to 0.05 were considered to reflect weak evidence of an association.

The target sample size for the preferences substudy was 100 patients, in order to provide $95 \%$ confidence intervals for 
proportions of no wider than $\pm 10 \%$. Clinicians were harder to recruit than expected with a total of 44 participating in the preferences substudy.

\section{RESULTS}

The preferences substudy included 83 of the 122 women recruited to PORTEC from ANZGOG sites. The main reason for the discrepancy was due to the preferences substudy opening after the parent PORTEC-3 trial at all sites. Baseline characteristics of the participating patients are summarised in Table 1 . Their median age was 60 (range 36-77), and most were married (65\%) without dependent children (89\%), and expected to have support all or most of the time during ACT (72\%). Their cancers were most frequently FIGO 2008 stage IIIC disease (43\%), endometrioid histology (70\%), and treated with TAH-BSO surgery (81\%).

Characteristics of the 44 participating clinicians are summarised in Table 2. Their median age was 43 years (range $30-63$ ), and most were female $(56 \%)$ and married $(86 \%)$ with dependent children (81\%). There were more radiation oncologists (39\%) than gynaeoncological surgeons $(30 \%)$ or medical oncologists $(25 \%)$ with most clinicians having over 5 years of experience as a specialist (84\%).

Patients' expectations of HRQL. Patients' ratings at baseline of their expected HRQL during ACT are ranked in Table 3. Alopecia, nausea, and fatigue were the three symptoms expected to be most troublesome during ACT; appetite, energy, and mood were the three aspects of well-being expected to be worst during ACT. Baseline ratings on the HADS were indicative of moderate to severe anxiety in 19 of the 82 women (23\%) and moderate to severe depression in four women (5\%).

Patients' preferences for ACT. Patients' preferences for ACT at baseline (before experiencing it) are summarised in Figure 1. The median survival benefit reported for each scenario was the minimum benefit judged sufficient to make ACT for endometrial cancer worthwhile by $50 \%$ or more of the participants. The median survival benefits were an extra 1 year in survival time beyond baselines of 5 and 8 years without ACT, and an extra 5 and $4 \%$ in survival rates beyond baselines of 50 or $65 \%$ respectively at 5 years without ACT. The preferences of patients varied widely over the entire range of options from no extra benefit to an extra 15 years in survival time and an extra $50 \%$ in survival rates.

At baseline, patients' preferences did not differ for the two survival time scenarios, but patients needed larger survival rate benefits necessary to make ACT worthwhile for the baseline survival rate of 50 vs $65 \% \quad(P<0.001)$. Patients' preferences changed over time (from baseline to 9 months) for the survival time scenarios, but not survival rate scenarios, according to whether they had ACT or not. Patients who had ACT judged smaller benefits sufficient over time whereas patients who had radiotherapy alone judged larger benefits necessary over time (change in median benefit - 3 months vs 2.5 months, $P=0.028$ ). Patients judging larger benefits necessary to make ACT worthwhile was weakly predicted by them being married or in a de facto relationship $v s$ being separated, single or widowed (median benefits of 1 year vs 6 months, $P=0.02$ and 6 vs $4 \%, P=0.02$ ).

Clinicians' preferences for ACT. Clinicians' preferences for ACT are summarised in Figure 2. The median survival benefits clinicians judged sufficient to make ACT worthwhile were an extra 1 year of survival time beyond a baseline of 5 or 8 years without ACT, and an extra 7 and $10 \%$ in survival rate beyond baselines of 50 or $65 \%$ respectively at 5 years without ACT. Clinicians' judged smaller benefits necessary to make ACT worthwhile for the baseline survival time of 5 vs 8 years $(P=0.03)$, but their preferences were similar for the survival rate scenarios. Clinicians judging larger benefits necessary to make ACT worthwhile was weakly predicted by clinicians having people dependent on their support at home $v s$ not (median benefits 15 vs 8 months; $P=0.02$ and 10 vs 5\%; $P=0.02)$.

Compared to patients, clinicians judged similar survival time benefits necessary to make ACT worthwhile (median benefits of 1 year vs 11 months, $P=0.4$ ) but needed larger survival rate benefits to make ACT worthwhile (median benefit 8.5 vs 5\%, $P=0.03$ ). Clinicians' preferences also varied over a narrower range

Table 1. Baseline characteristics of patients, their tumours, and treatments

\begin{tabular}{|c|c|c|}
\hline Characteristic & $N=83$ & $\%$ \\
\hline \multicolumn{3}{|l|}{ Age } \\
\hline Median (range) & \multicolumn{2}{|c|}{60 (36 to 77 ) years } \\
\hline \multicolumn{3}{|l|}{ Marital status } \\
\hline Married or de facto & 53 & 63.9 \\
\hline Separated or divorced & 18 & 21.7 \\
\hline Single & 7 & 8.4 \\
\hline Widowed & 4 & 4.8 \\
\hline Missing & 1 & 1.2 \\
\hline \multicolumn{3}{|l|}{ Dependent people at home } \\
\hline Yes & 16 & 19.3 \\
\hline \multicolumn{3}{|l|}{ Dependent children at home } \\
\hline Yes & 9 & 10.8 \\
\hline \multicolumn{3}{|l|}{ Highest level of education } \\
\hline Less than high school & 28 & 33.7 \\
\hline High school or trade qualification & 30 & 36.1 \\
\hline University or college degree & 24 & 28.9 \\
\hline Missing & 1 & 1.2 \\
\hline \multicolumn{3}{|l|}{ Employment status } \\
\hline Employed (full-time/part-time) & 56 & 67.5 \\
\hline Unemployed & 3 & 3.6 \\
\hline Retired & 23 & 27.7 \\
\hline Missing & 1 & 1.2 \\
\hline \multicolumn{3}{|c|}{ Friend or relative died of cancer } \\
\hline Yes & 63 & 75.9 \\
\hline \multicolumn{3}{|l|}{ Support available during $\mathrm{ACT}$} \\
\hline All of the time & 38 & 46.3 \\
\hline Most of the time & 21 & 25.6 \\
\hline Some of the time & 21 & 25.6 \\
\hline None of the time & 2 & 2.5 \\
\hline Missing & 1 & 1.2 \\
\hline \multicolumn{3}{|l|}{ Type of surgery } \\
\hline TAH-BSO & 68 & 81.9 \\
\hline TLH-BSO or LAVH-BSO & 15 & 18.1 \\
\hline \multicolumn{3}{|l|}{ Histology } \\
\hline Endometrioid & 58 & 69.9 \\
\hline Serous & 9 & 10.8 \\
\hline Clear cell & 6 & 7.2 \\
\hline Other & 10 & 12.1 \\
\hline \multicolumn{3}{|l|}{ FIGO 2008 stage } \\
\hline 1 & 15 & 18.1 \\
\hline ॥ & 11 & 13.3 \\
\hline IIIA & 13 & 15.3 \\
\hline IIIB & 4 & 4.8 \\
\hline IIIC & 35 & 42.2 \\
\hline Unknown & 5 & 6.0 \\
\hline \multicolumn{3}{|l|}{ Allocated treatment arm } \\
\hline Radiotherapy and ACT & 44 & 53.0 \\
\hline Radiotherapy & 39 & 47.0 \\
\hline \multicolumn{3}{|c|}{$\begin{array}{l}\text { Abbreviations: } \mathrm{ACT}=\text { adjuvant chemotherapy; } \mathrm{TAH}-\mathrm{BSO}=\text { Total Abdominal Hysterectomy- } \\
\text { Bilateral Salpingo-oophorectomy; TLH =Total Laparoscopic Hysterectomy; LAVH }= \\
\text { Laparoscopic-assisted vaginal hysterectomy. }\end{array}$} \\
\hline
\end{tabular}


Table 2. Clinicians' demographic characteristics

\begin{tabular}{|c|c|c|}
\hline Characteristic & $N=44$ & $\%$ \\
\hline \multicolumn{3}{|l|}{ Gender } \\
\hline Female & 24 & 54.5 \\
\hline \multicolumn{3}{|l|}{ Marital status } \\
\hline Married or de facto & 36 & 81.8 \\
\hline Separated or divorced & 3 & 6.8 \\
\hline Single & 3 & 6.8 \\
\hline Missing & 2 & 4.5 \\
\hline \multicolumn{3}{|l|}{ Dependent people } \\
\hline Yes & 25 & 56.8 \\
\hline \multicolumn{3}{|l|}{ Dependent children } \\
\hline Yes & 34 & 77.3 \\
\hline \multicolumn{3}{|c|}{ Friend or relative died of cancer } \\
\hline Yes & 35 & 79.5 \\
\hline \multicolumn{3}{|c|}{ Support available during $\mathrm{ACT}$} \\
\hline All of the time & 6 & 13.6 \\
\hline Most of the time & 11 & 25.0 \\
\hline Some of the time & 24 & 54.5 \\
\hline None of the time & 1 & 2.3 \\
\hline Missing & 2 & 4.5 \\
\hline \multicolumn{3}{|l|}{ Specialty } \\
\hline Medical oncologist & 11 & 25 \\
\hline Radiation oncologist & 17 & 39 \\
\hline Gynaeoncological surgeon & 13 & 30 \\
\hline Other & 3 & 3 \\
\hline
\end{tabular}

Table 3. Patients' ratings of expected health-related quality of life during adjuvant chemotherapy ranked by mean from worst affected to least affected

\begin{tabular}{|l|l|}
\hline \multicolumn{2}{|l|}{ Mean } \\
\hline Symptoms \\
\hline Hair loss & 5.6 \\
Fatigue & 5.0 \\
Nausea & 4.8 \\
Trouble sleeping & 4.1 \\
Diarrhoea & 3.9 \\
Anxiety & 3.9 \\
Vomiting & 3.8 \\
Irritability & 3.7 \\
Urinary symptoms & 3.5 \\
Altered sense of taste & 3.5 \\
\hline Global aspect of HRQL ${ }^{\text {b }}$ & \\
\hline Appetite & 5.8 \\
Energy & 5.9 \\
Mood & 6.4 \\
Physical well-being & 6.5 \\
Emotional well-being & 6.6 \\
Overall well-being & 6.7 \\
Mobility & 7.2 \\
\hline Abbreviation: HROL= Health-related quality of life. \\
a'Where $0=$ 'no trouble at all' up to 10='worst I can imagine'. \\
bWhere 0='worst possible' up to 10='best possible'. \\
\hline
\end{tabular}

(interquartile ranges (IQRs), 6 months to 1.5 years $v s 5$ months to 2 years, $P=0.0007$; and 5 to $10 \%$ vs 1 to $13 \%, P=0.004)$.

\section{DISCUSSION}

Decisions about ACT chemotherapy are difficult and involve a consideration of a patient's baseline risk of cancer recurrence, the benefit of ACT and the likely toxicities and inconveniences of the treatment. The benefits of ACT are usually modest and intangible manifesting as no recurrence of cancer over time. Toxicities, however, are real and frequent with potential lifelong consequences for patients who are otherwise well and free of cancer. Information that helps clinicians and patients better understand these aspects of decision-making about ACT, as in PORTEC-3, is much needed.

The main findings of our study are that most patients and clinicians judged moderate survival benefits sufficient to make ACT in addition to pelvic radiotherapy worthwhile in high risk or advanced endometrial cancer. Both patients' and clinicians' preferences varied over a wide range and were difficult to predict from their baseline characteristics.

This is the first published study of preferences for ACT in endometrial cancer. The median benefits judged sufficient to make ACT in endometrial cancer worthwhile in this study (an extra 1 year or 4 to $5 \%$ ) were larger than those required to make ACT worthwhile in breast cancer and colon cancer (an extra 1 day to 1 month, or an extra 0.1 to $1 \%$; Duric et al, 2005; Blinman et al, 2010), but similar to those required to make ACT worthwhile in NSCLC (9 months or 5\%; Blinman et al, 2015) and intra-peritoneal chemotherapy worthwhile in advanced ovarian cancer $(6$ months or 5\%; Blinman et al, 2013). The larger benefits required to make ACT worthwhile in endometrial cancer, compared with breast or colon cancer, may be determined by the older age of this patient groups: women with endometrial cancer are generally older and have fewer dependents than women with breast cancer (Blinman et al, 2010, 2015). Another key difference is that all patients in the current study had had pelvic radiotherapy whereas patients in our previous preferences studies only had chemotherapy. This receipt of radiotherapy may have skewed patients' preferences in this study, likely towards needing larger benefits to make ACT worthwhile.

We found that clinicians, compared with patients, had similar preferences in scenarios based on survival times (median benefit of 1 extra year), but required larger benefits in scenarios based on survival rate (median benefits of an extra 8.5 vs 5\%); clinicians' preferences also varied over a narrower range than those of patients. Earlier published studies mostly show that clinicians require substantially larger benefits than patients to make chemotherapy worthwhile. A widely cited study of preferences in advanced cancer, published more than 25 years ago, showed that the median 'chance of cure' needed to make a hypothetical regimen of chemotherapy worthwhile was $10 \%$ for oncologists but only $1 \%$ for patients with advanced cancer (Slevin et al, 1990). In our current study of preferences for ACT in endometrial cancer, the preferences of patients and clinicians were more similar, much as we found in our study of patients' and clinicians' preferences for ACT in NSCLC (Blinman et al, 2015), suggesting that contemporary clinicians' and patients' attitudes towards chemotherapy are more closely aligned. Possible reasons for this change over time may be a greater acceptance of chemotherapy as treatment for cancer due to improvements in efficacy, supportive care, and convenience and patients' expectations for cancer treatment more likely include ACT as a treatment option.

We found that patients' preferences changed over time for the survival time scenarios according to whether patients had had ACT or not. These results differed to our previous studies that found no change in patients' preferences over time (Blinman et al, 2013, 2015) and to Jansen et al's study in ACT for breast cancer where preferences were also stable over time (Jansen et al, 2001). These results of our current study should be interpreted with caution given the subgroup exploratory analysis, but possible explanations include patients' experience of ACT being better than expected or justification of their decision to participate in the trial and so be randomised to the ACT arm. 

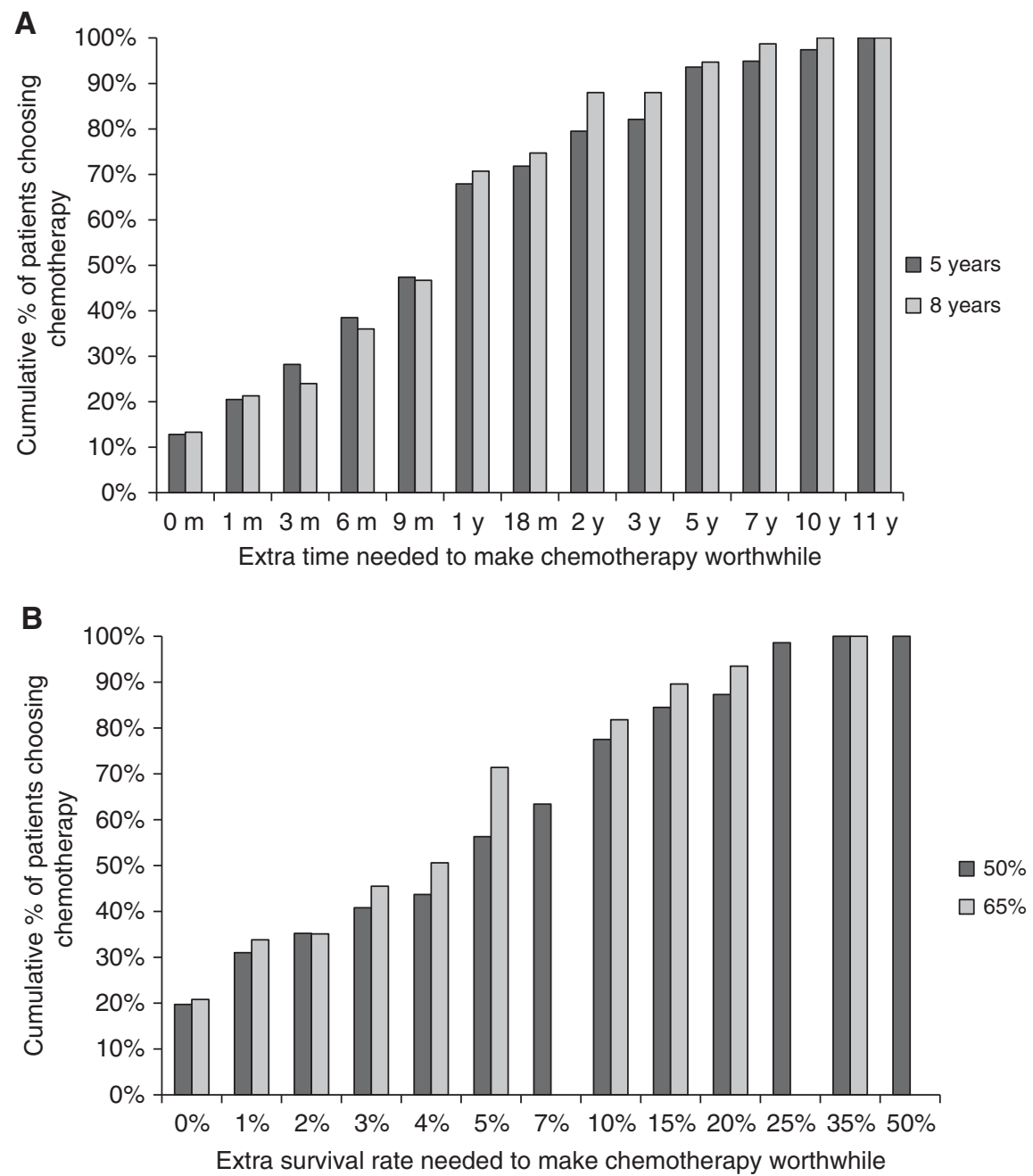

Figure 1. (A) Cumulative proportions of patients considering whether chemotherapy would be worthwhile for various improvements in 5- and 8 -year baseline survival times. (B) Cumulative proportions of patients considering whether chemotherapy would be worthwhile for various improvements in 50 and $65 \%$ baseline survival rates (at 5 years).

There were no strong predictors of either patients' or clinicians' preferences for ACT in endometrial cancer like in our previous studies. A systematic review found that the most consistent predictors of preferences were the benefit and toxicity of treatment, experience of treatment, and having dependents at home (Jansen et al, 2004). The lack of any significant predictors of preferences in this study might be a consequence of the sample size, but the consistency of this finding over our multiple studies supports the notion that preferences are not readily predictable from baseline characteristics, but are instead inherently individual and determined by one's experiences, values and attitudes, none of which are easily expressed, recognised or measured.

We nested this preferences substudy within a large, international, randomised phase III trial. The main advantages of this approach is that it ensures greater uniformity in the information provided to patients about the treatments in question, and that the preferences data are directly relevant to the treatment in the trial and so provide timely and important information to help clinicians and patients interpreting the outcome data from the trial. The PORTEC-3 trial, for example, was powered to detect a $10 \%$ absolute improvement in OS, which is substantially bigger than the median survival benefits of about $5 \%$ judged sufficient to make ACT worthwhile in the trial. Patients and clinicians will thus likely find ACT for endometrial cancer an acceptable treatment and readily adopt its use should the trial be positive.

Strengths of our current study are that it is the first study to determine patients' and clinicians' preferences for ACT for endometrial cancer, and doing this within the context of a randomised clinical trial as previously discussed which also allowed the recruitment of participants from a larger number and more geographically diverse centres. Limitations include selection bias of recruiting participants from a clinical trial who may have different preferences to patients who are not fit enough or willing to participate in a trial. The small sample size limits associations to only strong predictors of preferences of which there were none, consistent with our previous studies and the view that preferences are inherently individual. Recruitment to the preferences substudy was always determined by recruitment to the parent trial, but our final recruitment $(n=83)$ was not far from the estimated recruitment $(n=100)$. Recruitment of clinicians was far more difficult and well below that estimated. Informal feedback from sites included many clinicians uninterested in the study or not prioritising it as important, or declining because they felt uncomfortable answering questions about chemotherapy when this was not their usual treatment modality. Clinician engagement is clearly necessary for successful clinical 

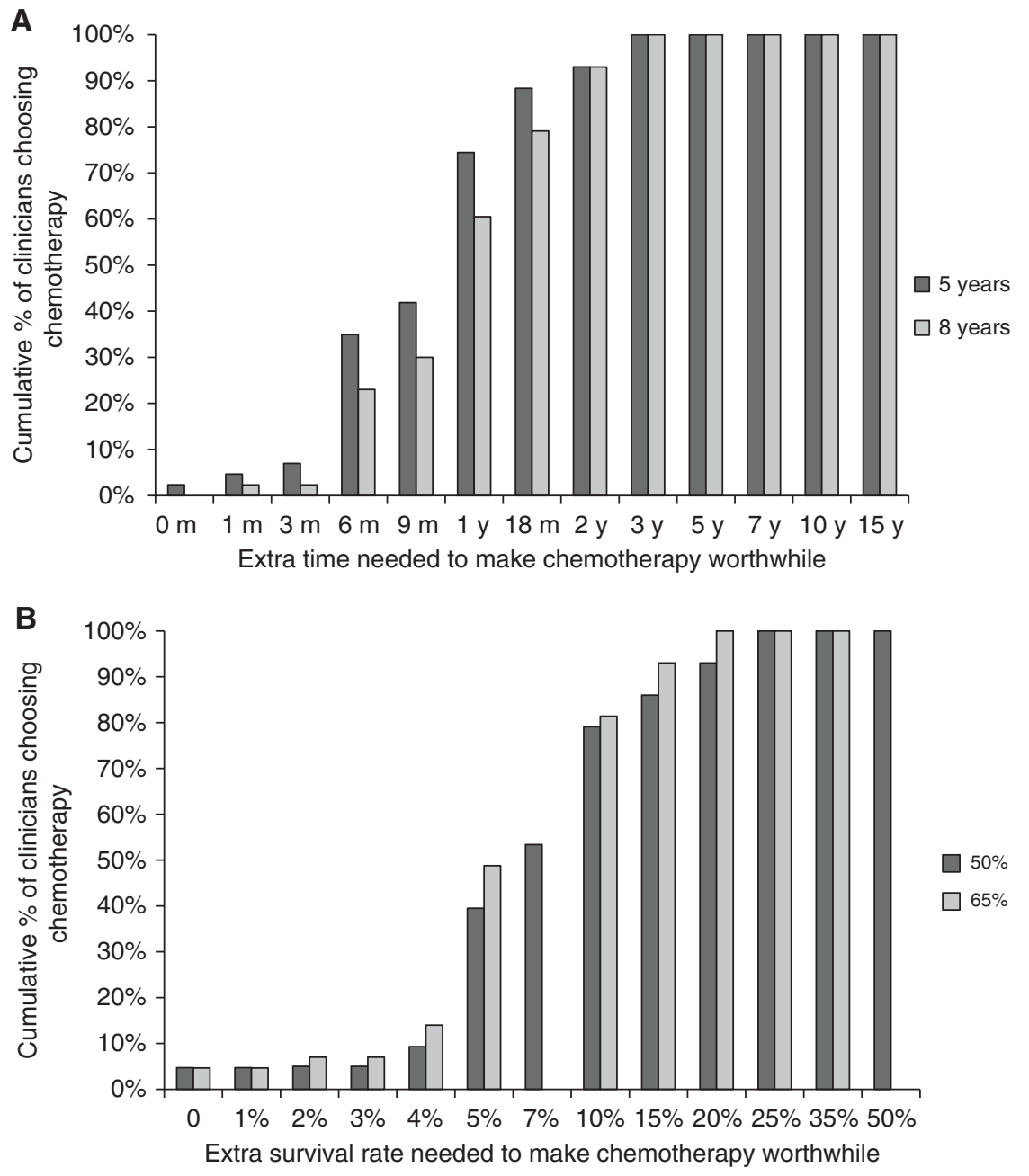

Figure 2. (A) Cumulative proportions of clinicians considering whether chemotherapy would be worthwhile for various improvements in 5 - and 8 -year baseline survival times. (B) Cumulative proportions of clinicians considering whether chemotherapy would be worthwhile for various improvements in 50 and $65 \%$ baseline survival rates (at 5 years).

research and our experience in this study will mean that we assess our methods in future studies.

Clinical implications of this study are that patients considering ACT for endometrial cancer have varying preferences, and therefore require careful, detailed discussions about the benefits and harms of chemotherapy to ensure their decisions are wellinformed and aligned with their personal views, attitudes, and priorities. Clinicians should appreciate that their views and recommendations about treatment are highly influential, explain the pros and cons of treatment; elicit patients' attitudes, values and priorities; and help formulate plans that are consistent with their patients' preferences, not just their own. Research implications are to establish the generalisability of these results by studying the preferences of women with endometrial cancer being treated in routine clinical practice as opposed to clinical trials; from other countries and cultural backgrounds; and, having different treatments.

In conclusion, patients and clinicians judged moderate survival benefits sufficient to make ACT worthwhile with pelvic radiotherapy for high-risk endometrial cancer in the PORTEC-3 trial. These benefits were larger than those judged sufficient by patients with breast or colon cancers, but similar to those judged sufficient by patients with lung or ovarian cancers. Preferences for ACT were highly variable, not easily predictable from baseline characteristics, and warrant careful elicitation and discussion as part of shared clinical decision-making.

\section{ACKNOWLEDGEMENTS}

This work was supported by NHMRC Project Grant 2009-2011 \#570894 and Cancer Australia Priority-driven Collaborative Cancer Research Scheme 2012-2015.

\section{CONFLICT OF INTEREST}

The authors declare no conflict of interest.

\section{REFERENCES}

Blinman P, Duric V, Nowak AK, Beale P, Clarke S, Briscoe K, Boyce A, Goldstein D, Hudson M, Stockler M (2010) Adjuvant chemotherapy for early colon cancer: what survival benefits make it worthwhile? Eur J Cancer 46: 1800-1807.

Blinman P, Gainford C, Donoghoe M, Martyn J, Blomfield P, Grant P, Kichenadasse G, Vaughan M, Brand A, Shannon C, Gebski V, Stockler M, Friedlander M (2013) Feasibility, acceptability and preferences for intraperitoneal chemotherapy with paclitaxel and cisplatin after optimal debulking surgery for ovarian and related cancers: an ANZGOG study. J Gynecol Oncol 24: 359-366.

Blinman P, Hughes B, Crombie C, Christmas T, Hudson M, Veillard A-S, Muljadi N, Millward M, Wright G, Flynn P, Windsor M, Stockler M, 
Mclachlan S-A (2015) Patients' and doctors' preferences for adjuvant chemotherapy in resected non-small-cell lung cancer: what makes it worthwhile? Eur J Cancer 51: 1529-1537.

Blinman P, King M, Norman R, Viney R, Stockler MR (2012) Preferences for cancer treatments: an overview of methods and applications in oncology. Ann Oncol 23(5): 1104-1110.

Duric VM, Stockler MR, Heritier S, Boyle F, Beith J, Sullivan A, Wilcken N, Coates AS, Simes RJ (2005) Patients' preferences for adjuvant chemotherapy in early breast cancer: what makes AC and CMF worthwhile now? Ann Oncol 16: 1786-1794.

Gibbons JD, Chakraborti S (2011) Nonparametric statistical inference. Springer: New York, NY.

Hogberg T, Signorelli M, De Oliveira CF, Fossati R, Lissoni AA, Sorbe B, Andersson H, Grenman S, Lundgren C, Rosenberg P, Boman K, Tholander B, Scambia G, Reed N, Cormio G, Tognon G, Clarke J, Sawicki T, Zola P, Kristensen G (2010) Sequential adjuvant chemotherapy and radiotherapy in endometrial cancer - results from two randomised studies. Eur J Cancer 46: 2422-2431.

Jansen SJ, Kievit J, Nooij MA, Stiggelbout AM (2001) Stability of patients' preferences for chemotherapy the impact of experience. Med Decis Making 21: 295-306.

Jansen SJT, Otten W, Stiggelbout AM (2004) Review of determinants of patients' preferences for adjuvant therapy in cancer. J Clin Oncol 22: 3181-3190.

Jemal A, Bray F, Center MM, Ferlay J, Ward E, Forman D (2011) Global cancer statistics. CA Cancer J Clin 61: 69-90.

Keys HM, Roberts JA, Brunetto VL, Zaino RJ, Spirtos NM, Bloss JD, Pearlman A, Maiman MA, Bell JG (2004) A phase III trial of surgery with or without adjunctive external pelvic radiation therapy in intermediate risk endometrial adenocarcinoma: a Gynecologic Oncology Group study. Gynecol Oncol 92: 744-751.

Maggi R, Lissoni A, Spina F, Melpignano M, Zola P, Favalli G, Colombo A, Fossati R (2006) Adjuvant chemotherapy vs radiotherapy in high-risk endometrial carcinoma: results of a randomised trial. $\mathrm{Br} J$ Cancer $\mathbf{9 5}$ : 266-271.

Morrow CP, Bundy BN, Homesley HD, Creasman WT, Hornback NB, Kurman R, Thigpen JT (1990) Doxorubicin as an adjuvant following surgery and radiation therapy in patients with high-risk endometrial carcinoma, stage I and occult stage II: a Gynecologic Oncology Group study. Gynecol Oncol 36: 166-171.

Nout RA, Van De Poll-Franse LV, Lybeert MLM, Wárlám-Rodenhuis CC, Jobsen JJ, Mens JWM, Lutgens LCHW, Pras B, Van Putten WLJ, Creutzberg CL (2011) Long-term outcome and quality of life of patients with endometrial carcinoma treated with or without pelvic radiotherapy in the post operative radiation therapy in endometrial carcinoma 1 (PORTEC-1) trial. J Clin Oncol 29: 1692-1700.

Randall ME, Filiaci VL, Muss H, Spirtos NM, Mannel RS, Fowler J, Thigpen JT, Benda JA (2006) Randomized phase III trial of whole-abdominal irradiation versus doxorubicin and cisplatin chemotherapy in advanced endometrial carcinoma: a Gynecologic Oncology Group Study. J Clin Oncol 24: 36-44.

Slevin ML, Stubbs L, Plant HJ, Wilson P, Gregory WM, Armes PJ, Downer SM (1990) Attitudes to chemotherapy: comparing views of patients with cancer with those of doctors, nurses, and general public. BMJ 300: 1458-1460.

Stockler MR, O'connell R, Nowak AK, Goldstein D, Turner J, Wilcken NRC, Wyld D, Abdi EA, Glasgow A, Beale PJ, Jefford M, Dhillon H, Heritier S, Carter C, Hickie IB, Simes RJ (2007) Effect of sertraline on symptoms and survival in patients with advanced cancer, but without major depression: a placebo-controlled double-blind randomised trial. Lancet Oncol 8: 603-612.

Susumu N, Sagae S, Udagawa Y, Niwa K, Kuramoto H, Satoh S, Kudo R (2008) Randomized phase III trial of pelvic radiotherapy versus cisplatinbased combined chemotherapy in patients with intermediate- and highrisk endometrial cancer: a Japanese Gynecologic Oncology Group Study. Gynecol Oncol 108: 226-233.

Torre LA, Bray F, Siegel RL, Ferlay J, Lortet-Tieulent J, Jemal A (2015) Global cancer statistics, 2012. CA Cancer J Clin 65: 87-108.

Zigmond AS, Snaith RP (1983) The hospital anxiety and depression scale. Acta Psychiatr Scand 67: 361-370.

This work is published under the standard license to publish agreement. After 12 months the work will become freely available and the license terms will switch to a Creative Commons AttributionNonCommercial-Share Alike 4.0 Unported License. 